\title{
OGLE-2013-SN-079: A LONELY SUPERNOVA CONSISTENT WITH A HELIUM SHELL DETONATION
}

\author{
C. Inserra ${ }^{1}$, S. A. Sim ${ }^{1}$, L. Wyrzykowski ${ }^{2,3}$, S. J. Smartit ${ }^{1}$, M. Fraser ${ }^{3}$, M. Nicholl ${ }^{1}$, K. J. Shen ${ }^{4}$, A. Jerkstrand ${ }^{1}$,

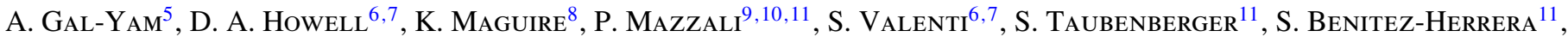 \\ D. Bersier ${ }^{9}$, N. Blagorodnova ${ }^{3}$, H. Campbell ${ }^{3}$, T.-W. Chen $^{1}$, N. Elias-Rosa ${ }^{10}$, W. Hillebrandt ${ }^{11}$, \\ Z. Kostrzewa-Rutkowska ${ }^{2}$, S. KozŁowski ${ }^{2}$, M. Kromer ${ }^{12}$, J. D. Lyman ${ }^{13}$, J. Polshaw ${ }^{1}$, F. K. RöPKe ${ }^{14}$, A. J. Ruiter ${ }^{15}$,

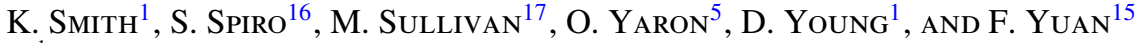 \\ ${ }^{1}$ Astrophysics Research Centre, School of Mathematics and Physics, Queens University Belfast, \\ Belfast BT7 1NN, UK; c.inserra@qub.ac.uk \\ ${ }^{2}$ University of Warsaw, Astronomical Observatory, Al. Ujazdowskie 400-478 Warszawa, Poland \\ ${ }^{3}$ Institute of Astronomy, University of Cambridge, Madingley Road, CB3 OHA Cambridge, UK \\ ${ }^{4}$ Department of Astronomy and Theoretical Astrophysics Center, University of California, Berkeley, CA 94720, USA \\ ${ }^{5}$ Benoziyo Center for Astrophysics, Weizmann Institute of Science, 76100 Rehovot, Israel \\ ${ }^{6}$ Las Cumbres Observatory Global Telescope Network, 6740 Cortona Drive, Suite 102 Goleta, CA 93117, USA \\ ${ }^{7}$ Department of Physics, University of California, Santa Barbara, Broida Hall, Mail Code 9530, Santa Barbara, CA 93106-9530, USA \\ ${ }^{8}$ European Southern Observatory for Astronomical Research in the Southern Hemisphere (ESO), Karl-Schwarzschild-Str. 2, \\ 85748 Garching b. Munchen, Germany \\ ${ }^{9}$ Astrophysics Research Institute, Liverpool John Moores University, Liverpool, UK \\ ${ }^{10}$ INAF - Osservatorio Astronomico di Padova, Vicolo dell'Osservatorio 5, I-35122 Padova, Italy \\ ${ }^{11}$ Max-Planck-Institut für Astrophysik, Karl-Schwarzschild-Str. 1, 85741 Garching, Germany \\ 12 The Oskar Klein Centre, Stockholm University, AlbaNova, SE-106 91 Stockholm, Sweden \\ ${ }^{13}$ Department of Physics, University of Warwick, Coventry CV4 7AL, UK \\ ${ }^{14}$ Institut für Theoretische Physik und Astrophysik, Universität Würzburg Emil-Fischer-Straße 31, D-97074 Würzburg, Germany \\ ${ }^{15}$ Research School of Astronomy \& Astrophysics, Mount Stromlo Observatory, The Australian National University Cotter Road, \\ Weston Creek, ACT 2611, Australia \\ ${ }^{16}$ Department of Physics (Astrophysics), University of Oxford, DWB, Keble Road, Oxford OX1 3RH, UK \\ ${ }^{17}$ School of Physics and Astronomy, University of Southampton, Southampton, SO17 1BJ, UK \\ Received 2014 October 22; accepted 2014 December 5; published 2015 January 9
}

\begin{abstract}
We present observational data for a peculiar supernova discovered by the OGLE-IV survey and followed by the Public ESO Spectroscopic Survey for Transient Objects. The inferred redshift of $z=0.07$ implies an absolute magnitude in the rest-frame $I$-band of $M_{I} \sim-17.6 \mathrm{mag}$. This places it in the luminosity range between normal Type Ia SNe and novae. Optical and near infrared spectroscopy reveal mostly $\mathrm{Ti}$ and $\mathrm{Ca}$ lines, and an unusually red color arising from strong depression of flux at rest wavelengths $<5000 \AA$. To date, this is the only reported SN showing Ti-dominated spectra. The data are broadly consistent with existing models for the pure detonation of a helium shell around a low-mass CO white dwarf and "double-detonation" models that include a secondary detonation of a $\mathrm{CO}$ core following a primary detonation in an overlying helium shell.
\end{abstract}

Key words: supernovae: general - supernovae: individual (OGLE-2013-SN-079) - surveys - white dwarfs

\section{INTRODUCTION}

The observational and physical parameter space of known supernova (SN) types has recently been expanded by the discovery of unusual optical transients. They are fainter and evolve on shorter timescales than normal Type Ia supernovae (SNe Ia) but are brighter than classical novae. Among them are bright and rapidly decaying objects like SN2002bj (Poznanski et al. 2010), SN2010X (Kasliwal et al. 2010), and slower evolving "Ca-rich gap" transients (Kasliwal et al. 2012) with $M_{R} \gtrsim-16 \mathrm{mag}$, of which SN2005E represents the prototype (Perets et al. 2010). These objects are characterized by strong $\mathrm{Ca}$ II features in their spectra, but their physical origin remains unexplained. It was initially suggested that some may be associated with the detonation of a helium layer on a lowmass CO white dwarf (WD). Helium outer layers may build up in binary systems in which a primary CO WD accretes from a degenerate (or semi-degenerate) He donor. If a sufficiently massive He layer is accreted $\left(\gtrsim 0.1 M_{\odot}\right)$, detonation may occur. In the case of a low-mass CO WD, this may lead to a faint thermonuclear $\mathrm{SN}$, roughly one-tenth the luminosity of a typical SN Ia luminosity, hence dubbed ".Ia" (Bildsten et al. 2007;
Shen \& Bildsten 2009; Shen \& Moore 2014). A detonation of the He layer may also trigger a secondary detonation of the $\mathrm{CO}$ core, in a so-called "double detonation" scenario (e.g., Fink et al. 2007, 2010; Kromer et al. 2010; Woosley \& Kasen 2011; Sim et al. 2012; Shen \& Bildsten 2014). Both scenarios show titanium and calcium dominated spectra, peak magnitudes of $-19 \lesssim M_{R} \lesssim-17$ together with rapid light curve declines (Shen \& Bildsten 2009; Sim et al. 2012).

However, since none of these transients have unambiguously matched the theoretical expectations of pure helium shell detonation or double detonation, alternative scenarios have also been proposed. A large explosion of a massive star with several $M_{\odot}$ of ejected mass may account for SN2010X and SN2002bj (Kleiser \& Kasen 2014). The same interpretation is suggested for the rapidly evolving Type Ic SN2005ek (Drout et al. 2013). Similarly, the helium shell detonation scenario was suggested for SN2005E (Perets et al. 2010; Waldman et al. 2011), but alternatives have been proposed for SN2005E and Ca-rich gap transients (see Kawabata et al. 2010; Valenti et al. 2014, and reference therein).

Here, we present the evolution of OGLE-2013-SN-079, a fast evolving SN with peak magnitude that lies in the luminosity 
Table 1

Observed Photometry of OGLE13-079 and Assigned Errors

\begin{tabular}{|c|c|c|c|c|c|c|c|c|}
\hline $\begin{array}{l}\text { Date } \\
\mathrm{dd} / \mathrm{mm} / \mathrm{yy}\end{array}$ & MJD & $\begin{array}{l}\text { Phase }^{a} \\
\text { (days) }\end{array}$ & $g$ & $V$ & $r$ & $I$ & $z$ & Telescope \\
\hline $16 / 09 / 13$ & 56551.15 & -14.0 & & & & $>22.48$ & & OGLE \\
\hline $20 / 09 / 13$ & 56555.18 & -10.0 & & & & $21.28(0.25)$ & & OGLE \\
\hline $24 / 09 / 13$ & 56559.15 & -6.0 & & & & $20.34(0.07)$ & & OGLE \\
\hline $27 / 09 / 13$ & 56562.16 & -3.0 & & & & $20.10(0.05)$ & & OGLE \\
\hline $30 / 09 / 13$ & 56565.19 & 0.0 & & & & $19.87(0.04)$ & & OGLE \\
\hline $03 / 10 / 13$ & 56569.32 & 4.1 & & $21.16(0.06)$ & & & & NTT \\
\hline $05 / 10 / 13$ & 56570.43 & 5.2 & $21.21(0.16)$ & & $20.58(0.03)$ & $19.99(0.04)$ & & FTS \\
\hline $06 / 10 / 13$ & 56571.16 & 6.0 & & & & $20.05(0.06)$ & & OGLE \\
\hline $06 / 10 / 13$ & 56571.42 & 6.2 & $21.41(0.12)$ & & $20.72(0.06)$ & $20.09(0.04)$ & & FTS \\
\hline $07 / 10 / 13$ & 56572.42 & 7.2 & $21.76(0.08)$ & & $20.77(0.02)$ & $20.16(0.04)$ & & FTS \\
\hline $07 / 10 / 13$ & 56573.03 & 7.8 & & $21.40(0.06)$ & & & & NTT \\
\hline $08 / 10 / 13$ & 56573.50 & 8.3 & $22.20(0.18)$ & & $20.86(0.07)$ & $20.09(0.10)$ & & FTS \\
\hline $09 / 10 / 13$ & 56574.44 & 9.2 & $22.41(0.20)$ & & $20.89(0.04)$ & $20.18(0.08)$ & & FTS \\
\hline $09 / 10 / 13$ & 56575.38 & 10.2 & & $21.71(0.05)$ & $20.99(0.06)$ & $20.37(0.06)$ & & NTT \\
\hline $10 / 10 / 13$ & 56575.50 & 10.3 & $22.69(0.23)$ & & $21.03(0.20)$ & $20.54(0.15)$ & & FTS \\
\hline $11 / 10 / 13$ & 56576.10 & 10.9 & & & & $20.45(0.08)$ & & OGLE \\
\hline $16 / 10 / 13$ & 56581.13 & 15.9 & & & & $21.06(0.20)$ & & OGLE \\
\hline $20 / 10 / 13$ & 56585.18 & 20.0 & & & & $21.00(0.24)$ & & OGLE \\
\hline $24 / 10 / 13$ & 56589.20 & 24.0 & & & & $21.34(0.26)$ & & OGLE \\
\hline $24 / 10 / 13$ & 56590.17 & 25.0 & & $22.51(0.16)$ & $21.98(0.14)$ & $21.63(0.16)$ & $21.36(0.16)$ & NTT \\
\hline $26 / 10 / 13$ & 56591.07 & 25.9 & & & & $>21.28$ & & OGLE \\
\hline $26 / 10 / 13$ & 56592.19 & 27.0 & & $22.62(0.08)$ & $22.09(0.05)$ & $21.86(0.08)$ & $21.67(0.18)$ & NTT \\
\hline $30 / 10 / 13$ & 56595.11 & 29.9 & & & & $21.99(0.34)$ & & OGLE \\
\hline $07 / 11 / 13$ & 56603.08 & 37.9 & & & & $>22.00$ & & OGLE \\
\hline $11 / 11 / 13$ & 56608.25 & 43.1 & $>23.40$ & & $23.20(0.13)$ & $22.65(0.17)$ & $22.35(0.17)$ & NTT \\
\hline \multicolumn{9}{|c|}{ Deepest limits } \\
\hline $25 / 11 / 13$ & 56622.15 & 57.0 & & $>23.45$ & & & & NTT \\
\hline $01 / 12 / 13$ & 56628.11 & 62.9 & $>23.50$ & & $>24.00$ & $>24.20$ & $>23.50$ & NTT \\
\hline $\begin{array}{l}\text { Date } \\
\mathrm{dd} / \mathrm{mm} / \mathrm{yy}\end{array}$ & MJD & $\begin{array}{l}\text { Phase }^{a} \\
\text { (days) }\end{array}$ & & $J$ & $H$ & $K$ & & Telescope \\
\hline $13 / 10 / 13$ & 56579.21 & 14.0 & & $19.98(.20)$ & $>19.60$ & $>19.20$ & & NTT \\
\hline $25 / 10 / 13$ & 56591.37 & 26.2 & & $>20.10$ & & & & NTT \\
\hline $02 / 11 / 13$ & 56599.31 & 34.1 & & $>20.00$ & & & & NTT \\
\hline
\end{tabular}

Note. ${ }^{\text {a }}$ Phase with respect to the $I$-band maximum.

window $-19 \lesssim M_{\mathrm{R}} \lesssim-15$. It differs from previous transients in showing titanium-dominated spectra and it appears to be a very promising helium detonation candidate.

\section{OBSERVATIONS}

OGLE-2013-SN-079 (hereafter OGLE13-079) was discovered by the OGLE-IV Transient Detection System (Wyrzykowski et al. 2014), at $m_{I} \sim 21.3 \mathrm{mag}$, on 2013 September 30.18 (magnitudes are reported in Table 1). The object coordinates have been measured on our astrometrically calibrated images: $\alpha=00^{\mathrm{h}} 35^{\mathrm{m}} 10.31 \pm 0.05, \delta=$ $-67^{\circ} 41^{\prime} 08^{\prime \prime} .51 \pm 0.05$ (J2000). A spectrum, taken at the New Technology Telescope (NTT) + EFOSC2 on October 4.33 UT, as part of the Public ESO Spectroscopic Survey for Transient Objects (PESSTO) ${ }^{18}$ showed a red continuum with broad lines similar to Type I SNe (Chen et al. 2013). The Galactic reddening toward the SN position is $E(B-V) \sim 0.02$ mag (Schlafly \& Finkbeiner 2011). Since the available spectra do not show Na ID lines related to internal reddening we will assume that the total reddening is given by the Galactic contribution.

18 www.pessto.org

\subsection{Host and SN location}

On October 16.21 UT, we took PESSTO spectra of the two elliptical galaxies, probably interacting, at coordinates $\alpha=00^{\mathrm{h}} 35^{\mathrm{m}} 04.47, \delta=-67^{\circ} 41^{\prime} 02^{\prime \prime} .7$ (triangle in Figure 1) and $\alpha=00^{\mathrm{h}} 35^{\mathrm{m}} 05^{\mathrm{s}} .21, \delta=-67^{\circ} 41^{\prime} 14^{\prime \prime} .7$ (square in Figure 1), finding redshifts $z=0.074$ and $z=0.072$, respectively. The second is also listed in the NASA/IPAC Extragalactic Database as 2MASXJ00350521-6741147 at redshift $z=0.071$. If one of the two galaxies is the host, the transient would be at a projected distance of $\sim 49.9 \mathrm{kpc}\left(\sim 35^{\prime \prime}\right)$ from the first galaxy, or $\sim 40.2 \mathrm{kpc}$ $\left(\sim 30^{\prime \prime}\right)$ from the second.

In our deepest images $(\mathrm{VrI})$, we performed aperture photometry on the possible host (the closest). We found that the radius containing half of the light of the galaxy is $R_{1 / 2} \sim 28 \mathrm{kpc}$, similar to the value $R_{1 / 2} \sim 30 \mathrm{kpc}$ measured using the $V$ magnitude of the galaxy and a Sérsic index of $n=4$ (Graham 2013, and reference therein). Thus OGLE13-079 is likely located in the extreme outskirts of the host, in the halo region. Based on this identification, we adopt $z=0.07$ as the redshift for OGLE13079 throughout the following.

The host galaxy type and the remote location of OGLE13-079 would argue against a massive star origin of this explosion (Hamuy et al. 1996; Anderson \& James 2009). 

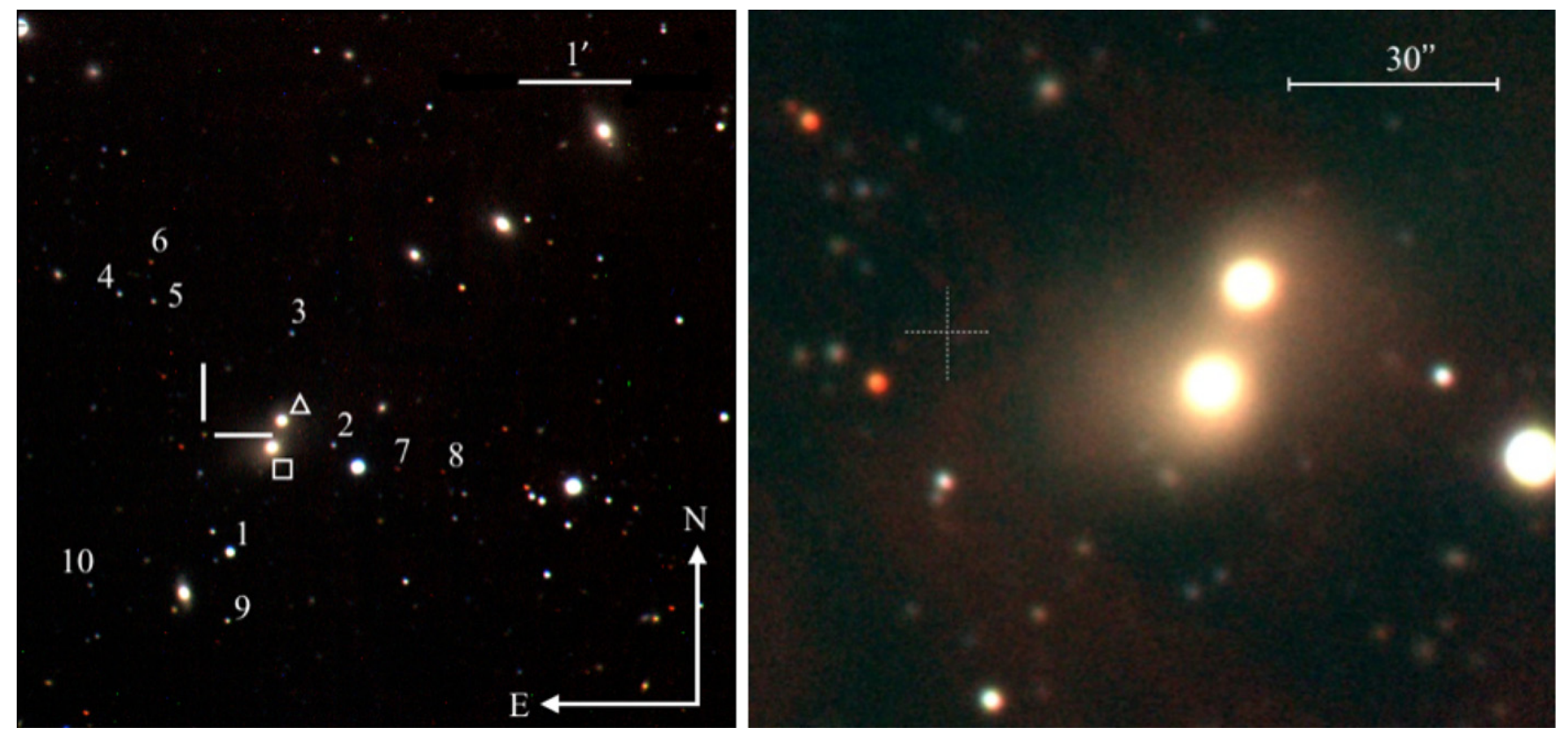

Figure 1. Left: FTS+Merope $+g / r / i$ image of OGLE13-079 (cross marks). The sequence of stars in the field is indicated. The two closest galaxies to OGLE13-079 are indicated (square and triangle). Right: NTT+EFOSC2 $+g / r / i$ image zoom of OGLE13-079 position (dashed cross). The reddish pattern is due to strong fringing of the $i \# 705$ filter

Table 2

Spectroscopic Observations

\begin{tabular}{lccccc}
\hline \hline $\begin{array}{l}\text { Date } \\
\text { dd/mm/yy }\end{array}$ & MJD & $\begin{array}{c}\text { Phase }^{\mathrm{a}} \\
(\text { days })\end{array}$ & $\begin{array}{c}\text { Range } \\
(\AA)\end{array}$ & $\begin{array}{c}\text { Resolution } \\
(\AA)\end{array}$ & $\begin{array}{c}\text { Instrumental } \\
\text { Configuration }\end{array}$ \\
\hline 03 Oct 13 & 56569.32 & 4.1 & $3700-9300$ & 18 & NTT+EFOSC2+gm13 \\
07 Oct 13 & 56573.05 & 7.9 & $3400-10300$ & $11 / 16$ & NTT+EFOSC2+gm11/gm16 \\
15 Oct 13 & 56580.18 & 15.0 & $3400-23200$ & $0.6 / 2$ & VLT+XSHOOTER+VIS/NIR \\
30 Oct 13 & 56596.08 & 30.9 & $3400-23200$ & $0.6 / 2$ & VLT+XSHOOTER+VIS/NIR \\
\hline
\end{tabular}

Note. ${ }^{\text {a }}$ Phase with respect to the $I$-band maximum.

The offset distribution of 520 SNe published by Kasliwal et al. (2012) supports this idea. Massive stars could reach large distances as a consequence of tidal stripping during galaxy interaction but they should then be in the intracluster environment of tidal tails. We do not observe such tails in our data.

We note that the host and location of OGLE13-079 are different from the bright and fast objects SNe 2002bj and 2010X, which both occurred close to the galaxy nuclei (Poznanski et al. 2010; Kasliwal et al. 2010). On the other hand, Ca-rich objects like SN2005E were found far from the nucleus (Kasliwal et al. 2012; Yuan et al. 2013; Lyman et al. 2014). OGLE13-079 shows the largest projected distance from its host nucleus among these types of objects and it is comparable only to PTF09dav (Sullivan et al. 2011).

\subsection{Data}

Optical and near infrared (NIR) images were reduced (trimmed, bias subtracted, and flat-fielded) using the PESSTO (Smartt et al. 2014), the OGLE (Wyrzykowski et al. 2014), and Faulkes Telescope pipelines. Photometric zero-points and color terms were computed using observations of standard fields (VI in Vega and $g r z$ in AB system). We then calibrated the magnitudes of a local stellar sequence shown in Figure 1. The average magnitudes of the local-sequence stars were used to calibrate the photometric zero-points in non-photometric nights. The JHK photometry was calibrated to the Two Micron All
Sky Survey system using the same local sequence stars. Our optical and NIR photometric measurements were performed using the point-spread function (PSF) fitting technique. Differences between passbands were taken into account (applying the S-correction; Stritzinger et al. 2002; Pignata et al. 2004).

All spectra (Table 2) were reduced and calibrated in the standard fashion (including trimming, overscan, bias correction, and flat-fielding) using standard routines within IRAF. The final flux calibration was checked by comparing the integrated spectral flux, transmitted through standard Sloan or Bessell filters, with our photometry. We applied a multiplicative factor when necessary, and the resulting flux calibration is accurate to within 0.2 mag.

\section{PHOTOMETRY}

\subsection{Light Curves}

OGLE13-079 was observed during the rising phase only in the $I$-band but subsequently followed in a range of optical filters until it disappeared beyond our detection threshold in late November (Figure 2). The non-detection four days before the first observation places a strong constraint on the explosion epoch, allowing us to define the rise time and the initial shape of the light curve. We estimate the explosion to have occurred at MJD $56553.5 \pm 2$, hence $\sim 11$ days before $I$-band maximum. The post-peak decline has the same shape in each band with 

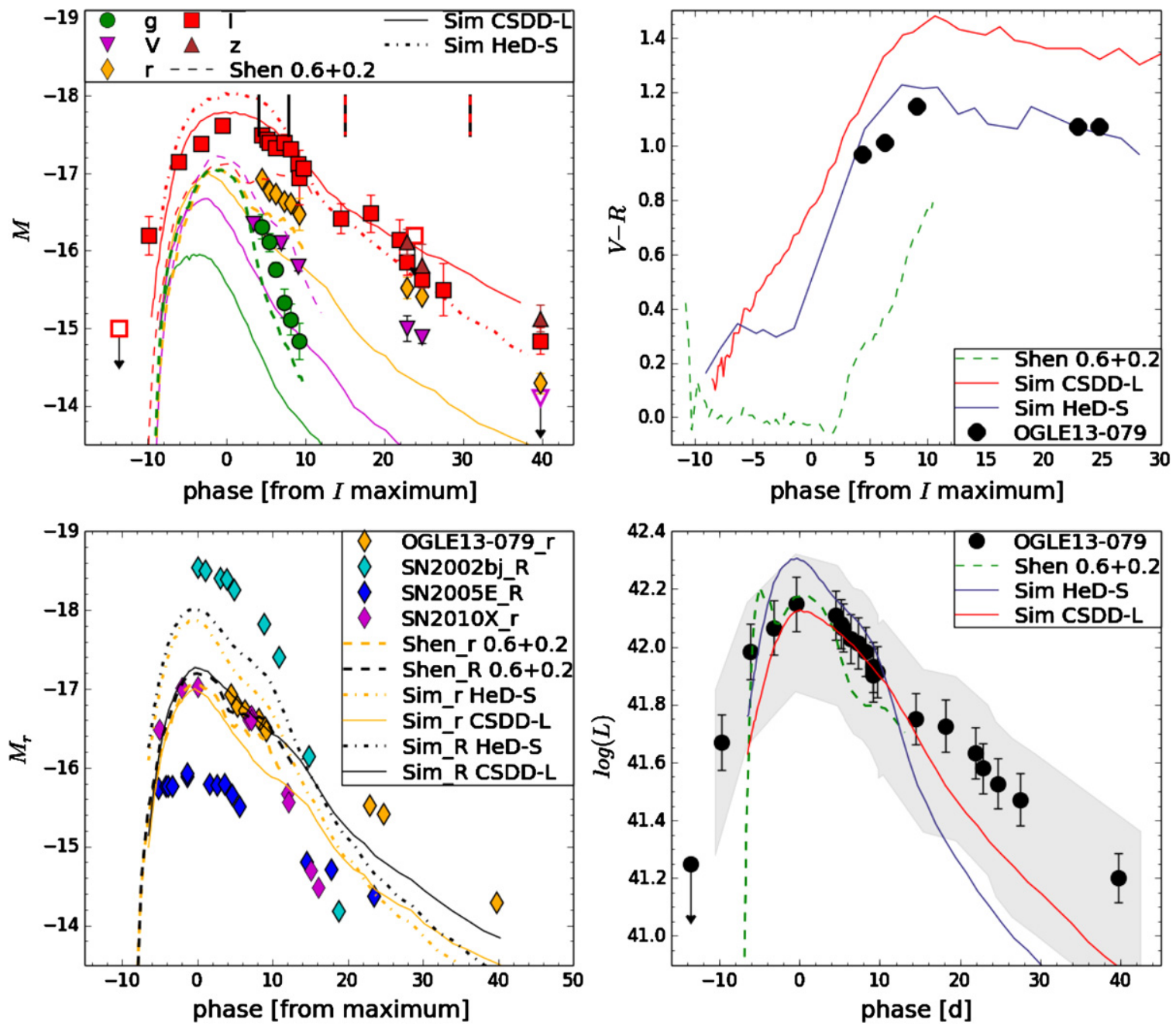

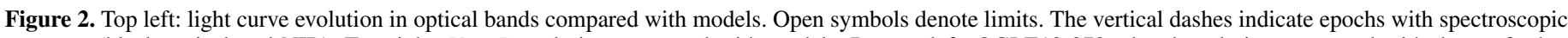

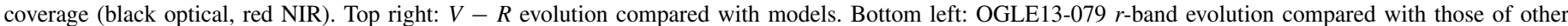

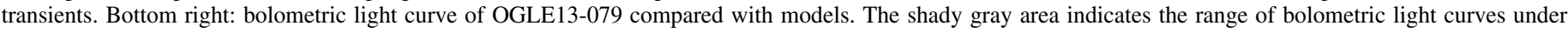
different assumptions.

the exception of the $g$ band, in which OGLE13-079 fades by $1.5 \mathrm{mag}$ over five days, in contrast to an average decrease of $0.48 \mathrm{mag}$ in the other bands. The slope in the $\mathrm{VrIz}$ bands is fairly constant until the last detection, with the exception of a shoulder in the $I$ band at $\sim 15$ days after peak that is reminiscent of the secondary maximum in type Ia (Kasen \& Plewa 2007).

The OGLE13-079 magnitudes are comparable to SN2010X but $\sim 1.4$ mag fainter than SN2002bj and $\sim 1.4$ mag brighter than SN2005E. Indeed, it is brighter than the most luminous $\left(M_{R}=-16.4 \mathrm{mag}\right.$ for PTF09dav, Kasliwal et al. 2012) of the Ca-rich transients. However, the post-peak decline of OGLE13-079 of $\Delta m_{15}(r) \sim 1.2$ is similar to that of SN2005E $\left(\Delta m_{15}(R) \sim 1.2\right)$ and slower than $\Delta m_{15}(R) \sim 2.4$ and $\sim 2.3$ of SN2002bj and SN2010X, respectively. Thus, while the OGLE13-079 $r$-band peak magnitude is similar to those of the fast evolving objects, the decline is comparable with the slower ones (Figure 2).

\subsection{Bolometric Light Curve}

To compare OGLE13-079 data with theoretical predictions from explosion models (see Section 3.3), a bolometric light curve of OGLE13-079 was constructed using similar methods to Inserra et al. (2013a, 2013b) and is shown in Figure 2. We initially built a $g$ VrIz pseudo-bolometric light curve and added a $u$ contribution by assuming $u-g \approx 0$, which is consistent with the synthetic photometry retrieved from our early spectra. We added a NIR contribution assuming $V-J \approx 1.8$ to be constant throughout the evolution and adopting NIR colors $(J-H$ and $J-K)$ similar to the average of $\mathrm{SNe}$ Ia (Contreras et al. 2010; Friedman et al. 2014). Clearly, the light curve constructed via these steps has substantial uncertainties, therefore, we also construct a robust lower limit on the bolometric light curve by integrating only over the measured flux in $g V r I z$. We placed an upper limit on the bolometric light curve by using the best-fit blackbody curve of the spectral energy distribution retrieved through the red optical bands $(r I z)$ by integrating from near ultraviolet to $K$-band.

The peak luminosity $L_{\text {bol }} \approx 1.4 \times 10^{42} \mathrm{erg} \mathrm{s}^{-1}$ is roughly a factor ten and two less than in normal and faint (SN1991bg-like) $\mathrm{SNe}$ Ia, respectively.

\subsection{Models and Theoretical Light Curves}

We compared our photometric and spectroscopic data with those predicted for the detonation of a $0.20 M_{\odot}$ He layer around 
a $0.60 M_{\odot} \mathrm{CO}$ core presented by Shen et al. (2010, hereafter $\left.0.6+0.2 M_{\odot}\right)$. We also compare to angle-averaged predictions from 2D models computed by Sim et al. (2012): specifically, we chose one of their helium shell detonations (HeD-S, which has a $0.59 M_{\odot} \mathrm{CO}$ core plus $0.21 M_{\odot}$ He layer) and one of their double detonations (CSDD-L, which has a $0.59 M_{\odot} \mathrm{CO}$ core plus $0.21 M_{\odot}$ He layer). These were chosen because they give the best match to OGLE13-079 absolute magnitude.

Of the models we considered, the best overall fit to the data is found with the CSDD-L model. However, the match is not perfect and, with the exception of the $I$ band, the model is always too faint. We note that a good match of the $I$-band evolution is also achieved by the HeD-S model but this model has a brighter and wider peak than the data or the CSDD-L model. The $0.6+0.2 M_{\odot}$ model of Shen et al. is fainter and has a faster decline compared to the HeD model, $\sim 8$-10d, depending on the band. This is likely a consequence of differences in the model ejecta structure and ionization state, which lead to differing degrees of line blanketing in the blue and reprocessing of the $\mathrm{UV} /$ blue light by heavy elements. The $0.6+0.2 M_{\odot}$ model does produce an $I$-band shoulder, although $\sim 10$ days earlier than observed. We note that the rise time of OGLE13-079 is similar to those predicted by the models considered, while the "late" ( $>15 \mathrm{~d}$ ) decline has a comparable slope to the Sim et al. the models. It is also noticeable that SNe 2002bj and 2010X are too rapidly fading compared to the models, while SN2005E is too dim.

The best match of the bolometric light curve is also found with the CSDD-L model, which is able to adequately fit the data from $-6 \mathrm{~d}$ to $16 \mathrm{~d}$ from maximum. After that, OGLE13-079 declines more slowly for the next 10 days $(15 \lesssim \mathrm{d} \lesssim 25)$ but then settles onto a decline slope similar to the model. The HeD-S model is between the limits of the bolometric light curves (gray area in Figure 2), but the fit generally appears poorer both around peak and at later times compared to the CSDD-L model. The Shen et al. model also fits the data reasonably well, although in the bolometric light curve of OGLE13-079 we do not observe any double-peaked behavior. Rise time and post maximum evolution differences between data and models are roughly similar to the single bands previously shown.

\subsection{Color Evolution Comparison}

In the top right panel of Figure 2 we compare the $V-R$ color evolution of OGLE13-079 with those predicted by the models. Since we did not have $R$-band observations, we transformed $r$ to $R$ magnitude using SNAKE (a PYTHON code for $K$-correction and magnitude conversion; $C$. Inserra, in preparation). Although the light curves are more similar to the CSDD-L model (see above), the $V-R$ behavior is closer to the HeD-S model with a $V-R \sim 1.1$ after 10 days since maximum. We note that the $V-R$ evolution is also comparable to CSDD-L model but shifted by $\sim 0.3$ to bluer values. Both the Sim et al. and Shen et al. models of pure helium detonation have similar behavior, although the Sim et al. models are systematically redder. ${ }^{19}$

\section{SPECTROSCOPY}

The rest-frame spectra are shown in Figure 3 and reported in Table 2. The striking features of the spectral sequence are the red color and the two prominent absorption profiles at $5300 \AA$

\footnotetext{
19 The redder colors of the Sim et al. compared to Shen et al. models are likely attributable to a combination of differences in the treatment of ionization and ejecta composition.
}

and $5900 \AA$. To our knowledge, such features have never been observed at an early phase in any other SN. By comparison with wavelengths and oscillator strengths from the Kurucz database (Kurucz \& Bell 1995) we identified these as multiple, blended Ti lines. Similar lines have been predicted in helium shell detonation models (Shen et al. 2010; Waldman et al. 2011). The spectra are dominated by Ti II lines during the $30 \mathrm{~d}$ post-peak evolution; the majority of the line profiles bluer than $6000 \AA$ are related to Ti II lines, ${ }^{20}$ with the exception of the weak, or possibly absent, Ca H\&K lines. We note that sub-luminous type Ia also show strong Ti absorptions in the blue. The Ca II NIR triplet becomes more prominent from the second spectrum onward. However, it is weaker than the $5300 \AA$ line and weaker than typically observed in Ca-rich objects. In these objects the $\mathrm{Ca}$ II is roughly three times stronger than other features (see Valenti et al. 2014). The NIR absorption around $9800 \AA$ could be attributed either to Ti II line or Ca II. We do not observe any obvious lines associated with intermediate elements such as $\mathrm{Si}$, $\mathrm{S}$ and light elements as $\mathrm{Mg}, \mathrm{C}$ and $\mathrm{O}$ as seen in other possible helium shell detonation candidates. Ti II and $\mathrm{Ca}$ II are dominant, and consistent with what is expected in a helium shell detonation (see also Holcomb et al. 2013). We note that OGLE13-079 is the first transient showing strong Ti lines, hence it could be the first example of what might be called a "Ti-strong" object.

\subsection{Comparison with other Helium Shell Detonation Candidates}

In Figure 3 we show OGLE13-079 with three other well studied transients of similar luminosity, SNe 2002bj, 2005E, and 2010X, at similar epochs. The spectra of SN2002bj are quite different from those of OGLE13-079: SN2002bj has a much bluer continuum and does not show the strong Ti lines (around $5300 \AA$ and $5900 \AA$ ). SN2005E has a similar red color to OGLE13-079 but also does not show the two prominent Ti lines. In contrast, SN2005E shows a strong Ca II NIR triplet feature, forbidden [Ca II] 7291,7323 $\AA$, and unambiguous He I lines. These led Perets et al. (2010) to identify the object as a Type $\mathrm{Ib}$ and a Ca-rich transient. We do not see forbidden [Ca II] or any He I lines in OGLE13-079 spectra at comparable phases. OGLE13-079 has a similar color to SN2010X but the spectral features are again different. The spectra are particularly distinct between $5500 \AA$ and $7500 \AA$ where Na I, O I and Mg I are not present in OGLE13-079. SN2010X also shows a prominent Ca II NIR triplet, which is similar in strength to those of other Ca-rich events but is not visible in the OGLE13-079 spectrum with comparable intensity. We note that the OGLE13-079 Ca II NIR triplet velocity is $\sim 4000 \mathrm{~km} / \mathrm{s}$ slower than those of SNe 2005E and 2010X in the same phase. We conclude that OGLE13-079 does not closely resemble any of these objects in its spectral features or colors.

\subsection{Comparison with Double-detonation and Helium Shell Detonation Models}

In Figure 3 we compare the OGLE13-079 spectrum with Sim et al. and Shen et al. models at similar epochs $(\sim 18$ days from explosion). The flux computed from the model spectra has been scaled to match that of OGLE13-079. All lines and their absorption and emission strength are well reproduced by the Shen et al. $0.6+0.2 M_{\odot}$ model. The model is slightly bluer, as also highlighted by the color evolution. The CSDD-L

\footnotetext{
20 We note that titanium production could be important for the positron budget and $511 \mathrm{keV}$ emission (Perets 2014).
} 

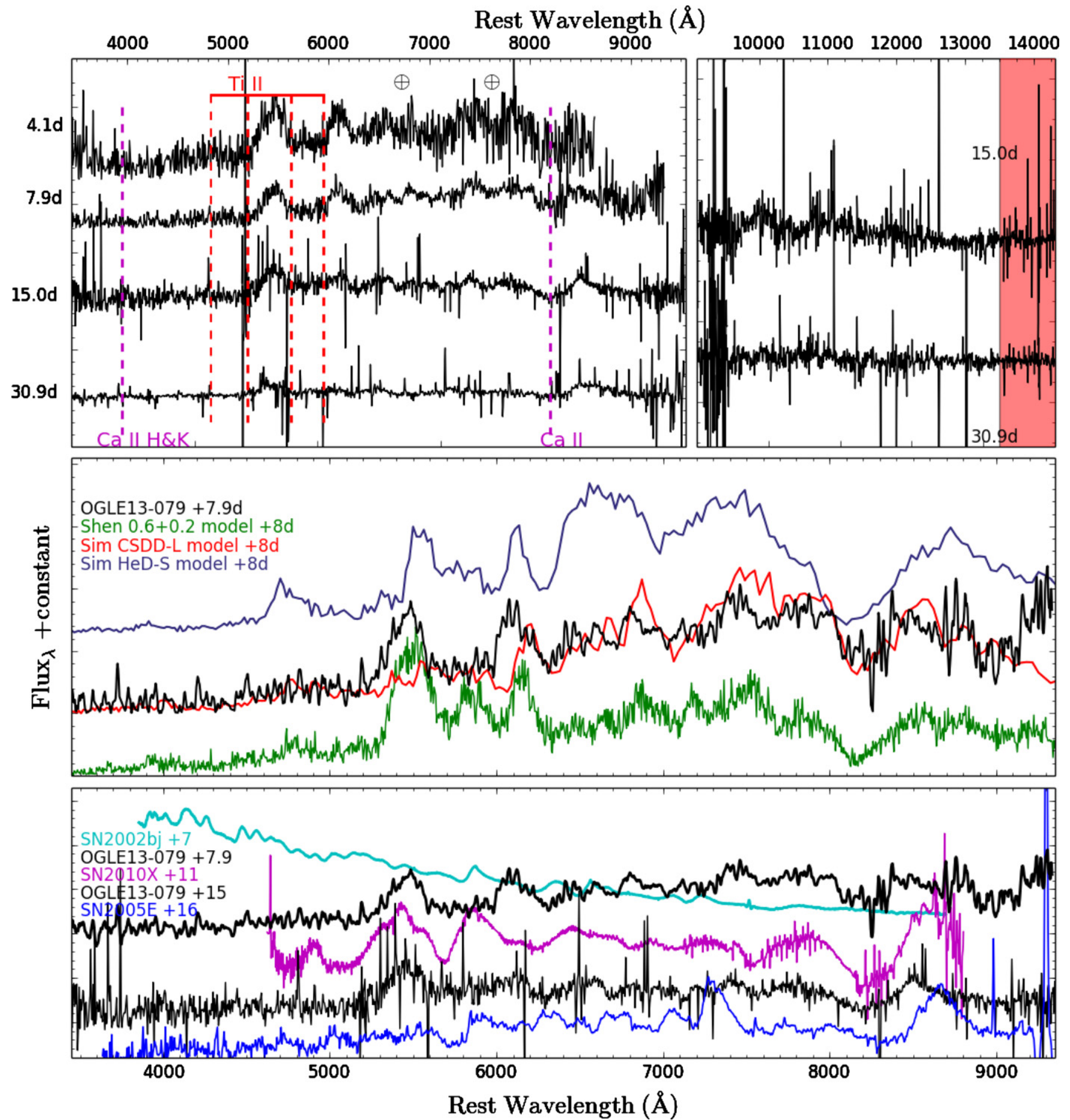

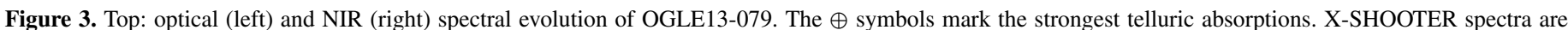

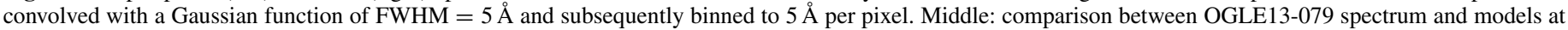
$\sim 8 \mathrm{~d}$ after $I$-band maximum ( $~ 18 \mathrm{~d}$ from explosion). Bottom: comparison between OGLE13-079 spectrum and other transients.

model does a reasonably good job in reproducing the spectrum, although we note that it does not provide a good match to the two noteworthy lines at $5300 \AA$ and $5900 \AA$, which we have attributed to Ti II. These differences between the models could be due to the distribution of $\mathrm{Fe}$-group elements in the ejecta, which are key to shaping the spectra (see Figure 6 of Sim et al. 2012), and generally to the treatment of ionization. The comparison between the $0.6+0.2$ and HeD-S models (similar masses but different densities) shows that the Sim et al. models fit the color better but the Shen et al. model fit the Ti II lines quite well. The existing models do not predict strong He lines, however, we note that further studies that include treatments of non-thermal excitation are required to better investigate the formation of He lines in the optical (Hachinger et al. 2012). We note that the majority of model lines are either not observed or have different strength (e.g., Ca II) in the other transients mentioned in Section 4.1. Although OGLE13-079 light curves could, in principle, be fitted with a massive star explosion it would be 
difficult to reproduce an oxygen-free and titanium-dominated spectral sequence.

\section{CONCLUSIONS}

OGLE13-079 is a transient in the luminosity region $-19 \lesssim$ $M_{\mathrm{R}} \lesssim-15$ spectrophotometrically different from similar transients discovered so far. Its maximum luminosity $M_{\mathrm{I}} \sim$ -17.6 is similar to SN2010X, thus brighter than the prototype Ca-rich transient SN2005E by $\sim 1.5 \mathrm{mag}$ and less luminous than SN2002bj by $\sim 1 \mathrm{mag}$. Its decline is comparable to that of SN2005E and other Ca-rich transients. Its location in the outskirts of the host galaxy ( $\sim 40 \mathrm{kpc})$, similar only to the Carich object PTF09dav, makes OGLE13-079 the most remote gap object so far discovered. OGLE13-079's spectral evolution is unique since there is little or no trace of $\mathrm{C}, \mathrm{O}, \mathrm{Mg}, \mathrm{Si}, \mathrm{S}$ in contrast to the other transients like SNe 2002bj, 2005E, and 2010X. The OGLE13-079 spectra are dominated by He-burning products such as Ti II and Ca II. The titanium lines are noticeably stronger than any other elements and suggests that OGLE13-079 could be the first "Ti-strong" transient.

OGLE13-079 is the first transient that reasonably well matches the synthetic observables predicted by models for detonation of a He layer around a low-mass CO WD and/or equivalent double detonation models. The strong lines of Ti II, a He-burning product, are reproduced by the nucleosynthetic reactions in these models. The lack of prominent features from intermediate-mass elements in the spectra of OGLE13-079 is striking. This combination is a quantitative signature of these explosion models. Not all the observed characteristics of OGLE13079 are well matched by these models. More focused and detailed theoretical simulations are warranted to investigate if the full data set can be matched with a pure helium shell detonation or a double detonation of a lower-mass shell $\left(<0.2 M_{\odot}\right)$.

C.I. thanks M.Kasliwal (SN2010X data). Based on observations at ESO as part of PESSTO (188.D-3003/191.D-0935/ 092.D-0555). Funded by FP7/2007-2013/ERC grant agreement [291222] (S.J.S.). We acknowledge support from STFC grant ST/L000709/1 (S.J.S., S.S.), TRR33 grant of DFG (S.T.), FP7/2007-2013 grant [267251] (N.E.R.), FP7/ERC grant [320360] (M.F.).

\section{REFERENCES}

Anderson, J. P., \& James, P. A. 2009, MNRAS, 399, 559

Bildsten, L., Shen, K. J., Weinberg, N. N., \& Nelemans, G. 2007, ApJL, 662, L95

Chen, T.-W., Inserra, C., Nicholl, M., et al. 2013, ATel, 5443, 1

Contreras, C., Hamuy, M., Phillips, M. M., et al. 2010, AJ, 139, 519

Drout, M. R., Soderberg, A. M., Mazzali, P. A., et al. 2013, ApJ, 774, 58

Fink, M., Hillebrandt, W., \& Röpke, F. K. 2007, A\&A, 476, 1133

Fink, M., Röpke, F. K., Hillebrandt, W., et al. 2010, A\&A, 514, A53

Friedman, A. S., Wood-Vasey, W. M., Marion, G. H., et al. 2014, arXiv: 1408.0465

Graham, A. W. 2013, in Planets, Stars and Stellar Systems. Vol. 6: Extragalactic Astronomy and Cosmology, ed. T. D. Oswalt \& W. C. Keel (Dordrecht: Springer), 91

Hachinger, S., Mazzali, P. A., Taubenberger, S., et al. 2012, MNRAS, 422, 70

Hamuy, M., Phillips, M. M., Suntzeff, N. B., et al. 1996, AJ, 112, 2391

Holcomb, C., Guillochon, J., De Colle, F., \& Ramirez-Ruiz, E. 2013, ApJ, 771,14

Inserra, C., Pastorello, A., Turatto, M., et al. 2013a, A\&A, 555, A142

Inserra, C., Smartt, S. J., Jerkstrand, A., et al. 2013b, ApJ, 770, 128

Kasen, D., \& Plewa, T. 2007, ApJ, 662, 459

Kasliwal, M. M., Kulkarni, S. R., Gal-Yam, A., et al. 2012, ApJ, 755, 161

Kasliwal, M. M., Kulkarni, S. R., Gal-Yam, A., et al. 2010, ApJL, 723, L98

Kawabata, K. S., Maeda, K., Nomoto, K., et al. 2010, Natur, 465, 326

Kleiser, I. K. W., \& Kasen, D. 2014, MNRAS, 438, 318

Kromer, M., Sim, S. A., Fink, M., et al. 2010, ApJ, 719, 1067

Kurucz, R., \& Bell, B. 1995, Atomic Line Data (Kurucz CD-ROM No. 23; Cambridge, MA: Smithsonian Astrophysical Observatory)

Lyman, J. D., Levan, A. J., Church, R. P., Davies, M. B., \& Tanvir, N. R. 2014, MNRAS, 444, 2157

Perets, H. B. 2014, arXiv:1407.2254

Perets, H. B., Gal-Yam, A., Mazzali, P. A., et al. 2010, Natur, 465, 322

Pignata, G., Patat, F., Benetti, S., et al. 2004, MNRAS, 355, 178

Poznanski, D., Chornock, R., Nugent, P. E., et al. 2010, Sci, 327, 58

Schlafly, E. F., \& Finkbeiner, D. P. 2011, ApJ, 737, 103

Shen, K. J., \& Bildsten, L. 2009, ApJ, 699, 1365

Shen, K. J., \& Bildsten, L. 2014, ApJ, 785, 61

Shen, K. J., Kasen, D., Weinberg, N. N., Bildsten, L., \& Scannapieco, E. 2010, ApJ, 715, 767

Shen, K. J., \& Moore, K. 2014, ApJ, 797, 46

Sim, S. A., Fink, M., Kromer, M., et al. 2012, MNRAS, 420, 3003

Smartt, S. J., Valenti, S., Fraser, M., et al. 2014, arXiv:1411.0299

Stritzinger, M., Hamuy, M., Suntzeff, N. B., et al. 2002, AJ, 124, 2100

Sullivan, M., Kasliwal, M. M., Nugent, P. E., et al. 2011, ApJ, 732, 118

Valenti, S., Yuan, F., Taubenberger, S., et al. 2014, MNRAS, 437, 1519

Waldman, R., Sauer, D., Livne, E., et al. 2011, ApJ, 738, 21

Woosley, S. E., \& Kasen, D. 2011, ApJ, 734, 38

Wyrzykowski, L., Kostrzewa-Rutkowska, Z., Kozlowski, S., et al. 2014, AcA, 64, 197

Yuan, F., Kobayashi, C., Schmidt, B. P., et al. 2013, MNRAS, 432, 1680 